Article

\title{
Precise Orbit Determination of FY-3C with Calibration of Orbit Biases in BeiDou GEO Satellites
}

\author{
Qiang Zhang ${ }^{1}{ }^{(1)}$, Xiang Guo ${ }^{1}$, Lizhong $Q u^{2, *}$ and Qile Zhao ${ }^{1,3, *}$ \\ 1 GNSS Research Center, Wuhan University, No. 129 Luoyu Road, Wuhan 430079, China; \\ qiangzhang@whu.edu.cn (Q.Z.); xiangguo@whu.edu.cn (X.G.) \\ 2 School of Geomatics and Urban Spatial Information, Beijing University of Civil Engineering and \\ Architecture, No. 15 Yongyuan Road, Beijing 102600, China \\ 3 Collaborative Innovation Center of Geospatial Technology, Wuhan University, No. 129 Luoyu Road, \\ Wuhan 430079, China \\ * Correspondence: qulizhong@bucea.edu.cn (L.Q.); zhaoql@whu.edu.cn (Q.Z.); Tel.: +86-027-6877-7227 (Q.Z.)
}

Received: 19 January 2018; Accepted: 26 February 2018; Published: 1 March 2018

\begin{abstract}
The emerging BeiDou navigation satellite system has contributed to global precise positioning and has recently moved toward space-borne applications. However, the contribution of BeiDou on LEO orbit determination applications is limited by the poor precision of the GEO satellite orbit and clock products. Current researches suggest that BeiDou GEO satellites should not be included in LEO precise orbit determination. Based on analyzing the characteristics of errors existing in BeiDou GEO orbit products, we propose a feasible method to mitigate the offsets in BeiDou GEO orbit errors by in-flight calibration of the systematic daily constant biases in the along-track and cross-track of BeiDou GEO satellites. The proposed method is investigated and validated using one entire month of onboard BDS data from the Chinese FY-3C satellite. The average daily RMS compared with the GPS-derived orbit indicates that our method achieves $6.2 \mathrm{~cm}$ three-dimensional precision. When compared to the solutions that disregard the GEO orbit errors scheme and roughly exclude the GEO scheme, the FY-3C orbit precision has been improved by $89.1 \%$ and $20.2 \%$, respectively. The average daily RMS values of phase residuals are about one centimeter for solutions that exclude GEO and that estimate systematic biases in GEO orbits. The calibrated orbits of GEO with the decimeter level in along-track and cross-track can be reconstructed by correcting the orbit biases estimated in the FY-3C precise orbit determination. Statistics of the FY-3C orbit quality, observation residuals, and precision of the recovered GEO orbits demonstrate that calibration of daily orbit biases in GEO can improve the precision of LEO orbit determination and enhance the reliability of the solution.
\end{abstract}

Keywords: precise orbit determination; FY-3C; orbit biases; BeiDou; GEO

\section{Introduction}

Precise orbit determination (POD) of the low Earth orbiter (LEO) is the primary prerequisite for a wide variety of scientific research and engineering applications, such as estimation of Earth gravity field, ionosphere sounding, ocean altimetry, Earth magnetic field geolocation, crustal deformation monitoring, atmospheric occultation sounding, establishment and maintenance of the terrestrial reference frame, and so on. GPS has been an efficient, convenient, and widely-employed technology for LEO POD since the 1980s. It has been validated in practice through post-analysis of space-borne GPS data obtained by GRACE [1], Jason-1 [2], GOCE [3,4], SWARM [5], and HY-2A [6] missions, etc. It has been demonstrated that centimeter level or even sub-centimeter radial orbit error is achievable by using accurate dual-frequency carrier-phase observations and satellite orbit dynamic models along with carefully-optimized empirical accelerations, which is also known as the reduced dynamic approach. 
In addition to GPS, a number of emerging global or regional satellite navigation systems are under development. The Chinese BeiDou navigation satellite system (BDS) has already provided excellent performance in precise positioning, estimating troposphere delay, and modelling Earth ionosphere, etc. principally in the Asian-Pacific region. As of July 2017, BeiDou consists of five geosynchronous Earth orbiters (GEO), six inclined geo-synchronization orbiters (IGSO), and three medium Earth orbiters (MEO). Furthermore, it will fully support global full-time and full-weather positioning, navigation, timing, and short message communication services until approximately 2020 [7].

The contributions of simulated and realistic onboard BDS observations on the LEO POD have already been investigated in some special aspects under different scenarios by a number of researchers. Chen et al. [8,9] presented the design overview of the navigation subsystem and the preliminary onboard performance of the space-borne BDS receiver experimental payload mounted on the LING QIAO satellite, which is the first Chinese LEO mobile communication experimental satellite. Liu et al. [10] analyzed the initial basic performance of the BeiDou regional navigation system used in the LEO absolute and relative orbit determination. They simulated space-borne receiver observations with the broadcast ephemeris of the BDS first phase and fitted reference orbits of GRACE and Jason-2 with different altitudes of about $500 \mathrm{~km}$ and $1330 \mathrm{~km}$, respectively. Xiong et al. [11] exhibited several qualitative results in analyzing satellite visibility, code multipath, ratio of cycle slips, post-orbit determination, single-point positioning, and real-time orbit determination, etc. by using one week of GPS and BDS tracking observations collected by the global navigation occultation sounder (GNOS) receiver equipped on the Chinese FengYun-3C (FY-3C) satellite [12-14]. They found that the differences in the average root mean square (RMS) of the 6-h orbit overlap indicated that the precision of the FY-3C orbit derived by GPS and BDS is $3.8 \mathrm{~cm}$ and $22.0 \mathrm{~cm}$, respectively. The results of the experiment performed by Li et al. [15] also assessed and validated the POD performance of FY-3C using BDS observations during March 2015. The results indicated that the GPS derived precise orbit achieves a precision of $2.7 \mathrm{~cm}$ for the overlapping differences. The precision of the BDS derived orbit reaches $30.1 \mathrm{~cm}$, and it can be improved to $8.4 \mathrm{~cm}$ by excluding the measurements from GEO satellites. Zhao et al. [16] also investigated the possibility and potentiality of augmenting the BeiDou precise orbit and clock products using the FY-3C onboard GPS and BDS observations. They found the precision of the FY-3C orbit using BDS observations is about $15.8 \mathrm{~cm}$ and is $11.7 \mathrm{~cm}$ with BeiDou products derived only by ground data and integrated ground and onboard data, respectively.

Through the corresponding research, comparison, results, and analysis mentioned above, the feasibility, availability, and efficiency of the LEO POD using onboard BDS observations have been sufficiently proved. The LEO orbit quality is very susceptible to the amount of the available BeiDou observation data and to the poor accuracy of orbit and clock products for BeiDou satellites, especially for GEO. The simulated experiment demonstrated that the BeiDou GEO with orbit accuracy several times inferior to IGSO and MEO would still improve the orbit quality for GRACE-A [10]. The FY-3C POD using practical BDS observations measured by the onboard GNOS receiver finds that including GEO will significantly decrease the precision of the FY-3C orbit to several decimeters. Fortunately, the FY-3C orbit less than $10 \mathrm{~cm}$ level can be easily reached by rejecting GEO.

The contribution of BeiDou GEO to precise point positioning (PPP) $[17,18]$ represents a positive correction when involving GEO with the current orbit and clock quality of GEO [19]. In contrast to the rough exclusion of GEO satellites this study proposes a new data processing scheme which incorporates GEO satellites through in-flight calibration of GEO orbit errors on the basis of a former study. The numerical results indicate that the proposed method improves the precise orbit accuracy by $20.2 \%$ as compared to excluding the GEO satellites.

The structure of this paper is arranged as follows: In Section 2 we introduce the current BeiDou precise products we adopt, then we assess BeiDou orbit quality using day boundary discontinuity (DBD) indicators and analyze the characteristics of orbit biases in GEO. In Section 3 we design and describe three kinds of comprehensive POD configurations for FY-3C using BDS to validate the proposed assumption that orbit biases can be estimated in the POD process together with the LEO 
orbit. Then we give a brief introduction to the distribution of onboard BDS measurements collected by FY-3C. Then we validate the precision of the three kinds of final orbits for FY-3C by comparing them with the GPS-derived reference orbit. The results support the rationality and correctness of the orbit bias calibration method proposed in this paper. Phase observation residuals derived in the three orbit solutions are analyzed further to support the method. Finally, the quality of the reconstructed GEO orbits by calibration of daily systematic biases are evaluated with the outliers removed. The discussion and conclusions associated with the experimental results and analysis are organized in Sections 4 and 5 , respectively.

\section{BeiDou Orbit Error Analysis and Mitigation}

\subsection{Assessment of BeiDou Precise Orbit Products}

Obviously, the precise orbit and clock products of the BeiDou constellation play an essential role in the accuracy of the BeiDou ranging observation models of POD using BDS measurements. There are several analysis centers (ACs) in the Multi-GNSS Experiment (MGEX) [20] project that provide BeiDou precise products. In our research we employed the GBM final products, which are computed using observations tracked by global ground stations from the International GNSS Service (IGS) [21] and MGEX network at Geo Forschungs Zentrum (GFZ) data processing and analysis center [22]. During July 2017 for BeiDou satellites the GBM products provide daily precise orbits every 5 min and clock offsets every $30 \mathrm{~s}$. The daily static and kinematic PPP using only dual-frequency BDS observations with GBM products can accomplish average position errors of $2 \mathrm{~cm}$ and $17 \mathrm{~cm}$, respectively [23]. Consequently, the GBM products are robust enough to conduct experiments for the POD of FY-3C only with BDS observations.

DBDs of BeiDou orbits are introduced to evaluate the orbit quality. This is because the assessment method using orbit overlap differences is no longer applicable since only the middle day of the three-day arc length solution is published by GFZ. The missing satellite position at the last moment (also known as midnight) is obtained using high precision orbit prediction within a five minutes window. It is believed that the precision of propagated BeiDou satellite orbits can achieve several centimeters over such a short time interval.

The statistical RMS of BeiDou orbit DBD series during July 2017 are listed in Table 1. The 3D RMS values of DBDs for GEO, IGSO, and MEO are within 1.66-8.36 m, 0.16-0.31 m, and 0.17-0.18 m, respectively. This shows that the orbit consistencies of BeiDou GEO are several times worse than those of IGSO and MEO.

Table 1. RMS of DBDs in along-track, cross-track, radial, and 3D for GBM orbits during July 2017.

\begin{tabular}{cccccc}
\hline \multirow{2}{*}{ Sat. ID } & & \multicolumn{3}{c}{ RMS of Orbit Day Boundary Discontinuities $(\mathbf{m})$} \\
\cline { 3 - 6 } & & Along-Track & Cross-Track & Radial & 3D \\
\hline \multirow{6}{*}{ GEO } & C01 & 1.10 & 1.24 & 0.09 & 1.66 \\
& C02 & 1.76 & 1.02 & 0.83 & 2.20 \\
& C03 & 1.59 & 1.23 & 1.16 & 2.32 \\
& C04 & 4.71 & 2.83 & 1.96 & 5.83 \\
& C05 & 7.87 & 0.88 & 2.67 & 8.36 \\
\hline \multirow{6}{*}{ IGSO } & C06 & 0.14 & 0.07 & 0.10 & 0.19 \\
& C07 & 0.12 & 0.26 & 0.12 & 0.31 \\
& C08 & 0.09 & 0.06 & 0.11 & 0.16 \\
& C09 & 0.14 & 0.07 & 0.21 & 0.26 \\
\multirow{3}{*}{ MEO } & C10 & 0.08 & 0.11 & 0.20 & 0.25 \\
& C13 & 0.06 & 0.10 & 0.13 & 0.17 \\
\hline & C11 & 0.12 & 0.07 & 0.12 & 0.18 \\
& C12 & 0.14 & 0.07 & 0.05 & 0.17 \\
& C14 & 0.17 & 0.06 & 0.04 & 0.18 \\
\hline
\end{tabular}




\subsection{Characteristics of BeiDou GEO Orbit Errors}

The present orbits of GEO produced by using only ground tracking network always display evident systematic biases with respect to the true orbits. This is because the range errors caused by the time-invariant part of orbit errors projected onto the signal propagation direction cannot be separated completely from the one-way float phase ambiguity parameters, which are due to the nearly stationary relative geometric structures between GEO and the ground stations in one arc segment of the POD. However, it is fortunate that the systematic biases in the along-track, cross-track, and radial components of the orbital reference frame could be mostly constants if the GEO is observed continuously by evenly-distributed ground stations.

Another practical method to determine the BeiDou orbit accuracy is by comparing orbits among various BeiDou products generated by several independent ACs of MGEX. The properties in the orbit difference time series can represent the features of BeiDou GEO orbit determination to a certain degree. Figure 1 shows the orbit difference series for BeiDou C02 between the GBM and WUM [24] products which are considered at the same precision level during July 2017. The relatively significant and separate short lines shows the orbit differences in along-track and cross-track during one day. The feature of $\mathrm{C} 02$ orbit differences can support the assumption that the distinguishing constant offsets with slight variations occur among single-day solutions published by GFZ, WUM, and the other ACs. All of these solutions adopt a similar strategy for BeiDou orbit determination using only ground station data.

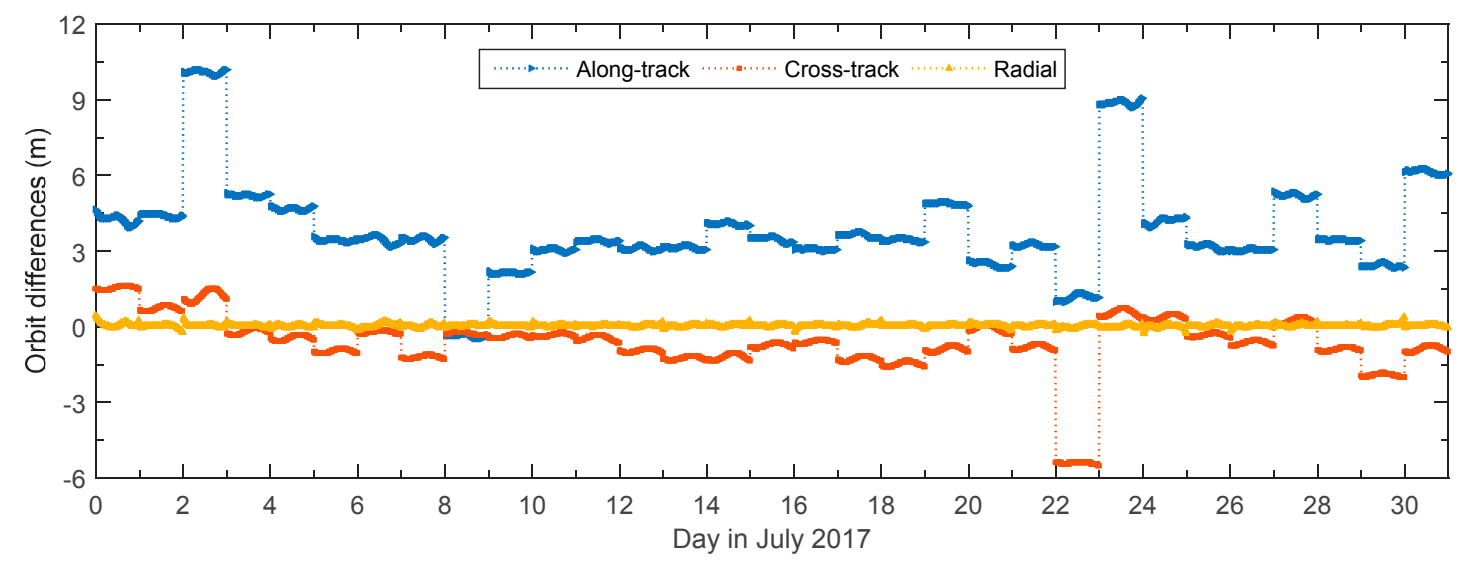

Figure 1. Orbit difference series in along-track, cross-track, and radial components with $15 \mathrm{~min}$ sampling interval for BeiDou C02 between the GBM and WUM orbit products during July 2017. Blue dots represent along-track, red dots represent cross-track, and yellow dots represent radial.

Daily jumps in the along-track and cross-track orbit differences are irregular and can reach a level of $10 \mathrm{~m}$. The daily orbit biases and DBDs are quite limited in the radial component of GEO orbits since the radial component of the orbits is tightly constrained by the accurate Earth gravity, which focuses on the gravitational direction from the satellite to Earth center. Orbit radial differences between GBM and WUM products represent a precision at the decimeter level and are not showed as daily constant offsets. It seems that orbit errors in the radial direction are greatly limited and the orbit biases most likely do not exist. Nevertheless, obvious biases in the radial component of GEO orbit products can be demonstrated by analyzing and assessing the residuals of independent satellite laser ranging (SLR) measurements $[23,24]$. Hence, the time period of POD for LEO should be limited to one daily arc. This avoids the situation where one continuous tracking arc containing the daily boundaries will be interrupted by interpolating the GEO satellite position at the time of signal sending. 


\subsection{Mitigation of BeiDou GEO Orbit Errors}

Reducing GEO orbit errors is similar to the phenomenon that errors in the orbit radial and along-track of the GPS can be mostly compensated by estimating the satellite clock offsets with a small size tracking station network. The small size is sufficient because of the small changes in the line-of-sight between whole stations and the satellite $[25,26]$. Furthermore, the systematic constant biases in GEO orbit products can still be absorbed mainly in the PPP of static or low kinematic stations by estimating phase ambiguity parameters owing to the almost unchanged line-of-sight direction. Errors in the GEO clock products are not considered separately due to the strong correlation between the satellite clock offsets and radial errors in orbits. Besides, there is a visible improvement in the positioning performance of PPP for ground stations in the Asia-Pacific region by including BeiDou GEO.

The batch least square processing strategy is adopted to adjust the estimated parameters in observation equations with the constraints of the LEO orbit dynamic models. The unknown parameters associated with the BeiDou phase observation equation for one GEO are expressed as Equation (1):

$$
\rho\left(t_{r}, t^{S}\right)=\rho\left(\boldsymbol{r}_{\mathbf{0}}, \boldsymbol{v}_{\mathbf{0}}, \boldsymbol{p}, \mathbf{P C O}, \delta t\left(t_{r}\right), A M B, d A, d C, d R\right)
$$

where $t_{r}$ and $t^{s}$ are the time of signal reception by receiver and the time of signal transmission by GEO transmitter, the symbol followed by $t_{r}$ or $t^{s}$ means that the physical quantity it represents changes over time $t_{r}$ or $t^{S}, \rho\left(t_{r}, t^{S}\right)$ is the phase observation of GEO measured by the onboard receiver, $\boldsymbol{r}_{\mathbf{0}}$ and $\boldsymbol{v}_{0}$ are the initial position and velocity vectors of the LEO center of mass at the reference time in a chosen conventional inertial frame, $\boldsymbol{p}$ is the parameter collection associated with the carefully selected dynamic models such as atmospheric drag, solar radiation pressure, and additional empirical forces, etc. PCO is the phase center offset vector of the LEO receiver antenna, $\delta t\left(t_{r}\right)$ is the LEO receiver clock offset at time $t_{r}, A M B$ is the constant phase ambiguity bias of the GEO in a tracking arc without cycle slips, $d A$, $d C$, and $d R$ denote the calibrated constant biases in the GEO orbit along-track, cross-track, and radial components, respectively, which represent the average differences between the GEO true reference orbit and the published orbit. More detailed considerations of the BeiDou observation models are listed in Table 2 of Section 3.1.

The partial derivatives of the carrier-phase and code pseudo-range observations with respect to the orbit biases of the GEO in the conventional inertial frame can be expressed as Equations (2) and (3):

$$
\begin{gathered}
\partial \rho\left(t_{r}, t^{S}\right)=\boldsymbol{e} \rho^{\mathrm{T}}\left(t_{r}, t^{S}\right)\left(\boldsymbol{e} A\left(t^{S}\right) \partial d A+\boldsymbol{e} C\left(t^{S}\right) \partial d C+\boldsymbol{e} R\left(t^{S}\right) \partial d R\right) \\
\boldsymbol{e} \rho\left(t_{r}, t^{S}\right)=\frac{\boldsymbol{R}\left(t^{S}\right)-\boldsymbol{r}\left(t_{r}\right)}{\left\|\boldsymbol{R}\left(t^{S}\right)-\boldsymbol{r}\left(t_{r}\right)\right\|} \\
\boldsymbol{e} C\left(t^{S}\right)=\frac{\boldsymbol{R}\left(t^{S}\right) \times \boldsymbol{V}\left(t^{S}\right)}{\left\|\boldsymbol{R}\left(t^{S}\right) \times \boldsymbol{V}\left(t^{s}\right)\right\|} \\
\boldsymbol{e} R\left(t^{S}\right)=\frac{\boldsymbol{R}\left(t^{S}\right)}{\left\|\boldsymbol{R}\left(t^{S}\right)\right\|} \\
\boldsymbol{e} A\left(t^{S}\right)=\boldsymbol{e C}\left(t^{S}\right) \times \boldsymbol{e} R\left(t^{S}\right)
\end{gathered}
$$

where $e \rho\left(t_{r}, t^{S}\right)$ is the unit vector from the receiver antenna to the GEO transmitter antenna, $e A\left(t^{S}\right)$, $e C\left(t^{S}\right)$, and $e R\left(t^{S}\right)$ are unit vectors parallel to the GEO orbit along-track, cross-track, and radial directions, respectively, which are used to convert the coordinates of constant offsets from the orbital reference frame to the conventional inertial frame, $d A, d C$, and $d R$ have the same physical unit as $\rho\left(t_{r}, t^{S}\right), \boldsymbol{R}\left(t^{S}\right)$ and $\boldsymbol{V}\left(t^{S}\right)$ denote the position and velocity vectors of the GEO in the inertial frame at time $t^{s}, r\left(t_{r}\right)$ is the position vector of receiver antenna in the inertial frame at time $t_{r}$.

Equation (2) indicates that the partial derivatives of range observations with respect to the GEO orbit biases can be expressed using $\boldsymbol{e} \rho^{\mathrm{T}}\left(t_{r}, t^{\mathcal{S}}\right) \boldsymbol{e} A\left(t^{S}\right), \boldsymbol{e} \rho^{\mathrm{T}}\left(t_{r}, t^{S}\right) \boldsymbol{e} C\left(t^{S}\right)$, and $\boldsymbol{e} \rho^{\mathrm{T}}\left(t_{r}, t^{S}\right) \boldsymbol{e} R\left(t^{S}\right)$, respectively. These variables represent the projective decomposition of the unit vector from receiver to satellite in the 
orbital reference frame, which are also known as the corresponding direction cosines. Consequently, the partial derivatives can be expressed as Equation (4):

$$
\begin{gathered}
\boldsymbol{e} \rho^{\mathrm{T}}\left(t_{r}, t^{S}\right) \boldsymbol{e} A\left(t^{S}\right)=\sin \theta\left(t_{r}, t^{S}\right) \cos \alpha\left(t_{r}, t^{S}\right) \\
\boldsymbol{e} \rho^{\mathrm{T}}\left(t_{r}, t^{S}\right) \boldsymbol{e} C\left(t^{S}\right)=\sin \theta\left(t_{r}, t^{S}\right) \sin \alpha\left(t_{r}, t^{S}\right) \\
\boldsymbol{e} \rho^{\mathrm{T}}\left(t_{r}, t^{S}\right) \boldsymbol{e} R\left(t^{S}\right)=\cos \theta\left(t_{r}, t^{S}\right)
\end{gathered}
$$

where $\theta\left(t_{r}, t^{s}\right)$ is the angle between the vector from receiver to satellite and the GEO orbit radial direction, it is also known as the zenith of line-of-sight from receiver to satellite, $\alpha\left(t_{r}, t^{s}\right)$ is the azimuth of vector from receiver to satellite in a planar coordinate system with base vector $e A\left(t^{s}\right)$ and $e C\left(t^{s}\right)$.

Equation (4) shows that the impact factors of orbit errors in the satellite orbital reference frame on range errors depend on the values of zenith $\theta\left(t_{r}, t^{s}\right)$ and azimuth $\alpha\left(t_{r}, t^{s}\right)$ of the line-of-sight. A negative impact factor means that the positive orbit error will cause the negative range error. An impact factor of one unit means that both the orbit error and the caused range error have the same size and sign. Figure 2 shows the series variation during 14 July 2017 of zenith and azimuth angles of the line-of-sight from positioning antenna of FY-3C GNOS receiver to transmitter antenna of BeiDou GEO. The zenith angle varies in the range of $0^{\circ}-10^{\circ}$ while the azimuth angle can vary in the range of $0^{\circ}-360^{\circ}$.

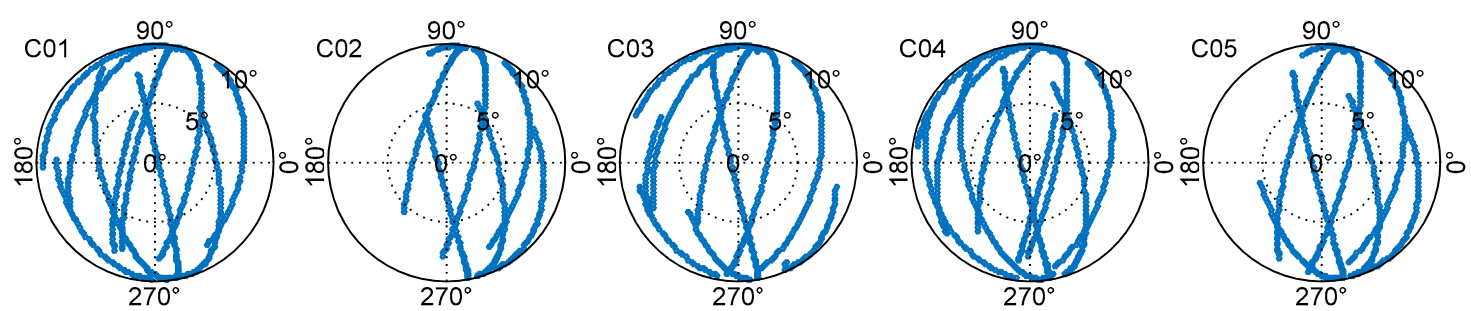

Figure 2. Zenith and azimuth series variation of vectors from FY-3C positioning antenna to the transmitter antenna of BeiDou GEO in the GEO orbital reference frame during 14 July 2017.

The corresponding partial derivatives of range observations with respect to the GEO orbit biases in the GEO orbital frame illustrate the sensitivity indicators of the range errors along the line-of-sight from FY-3C to the satellite on the specific orbit errors. By using the situation that the BeiDou C04 tracked by FY3C GNOS receiver during July 2017 as an example, Figure 3 shows the distribution for impact factors of orbit biases in along-track, cross-track, and radial on line-of-sight range errors from the satellite view of C04. As indicated in Figure 3, the coverage area on Earth where C04 can be observed by FY-3C is the service region of BeiDou C04 on FY-3C. It ranges in longitude from $80^{\circ} \mathrm{E}$ to $120^{\circ} \mathrm{W}$ and in latitude from $80^{\circ} \mathrm{S}$ to $80^{\circ} \mathrm{N}$. The central projection of the service region for BeiDou C04 is $160^{\circ} \mathrm{E}$ on the equator. This is very close to the theoretical values according to the maximum zenith $10^{\circ}$ for the average altitude $850 \mathrm{~km}$ of FY-3C.

The impact factors of C04 orbit biases on range errors show south-north, east-west, and central symmetries in orbit along-track, cross-track, and radial, respectively. The impact factors for the along-track and cross-track change within the range of -0.18 to 0.18 . The sensitivities of FY3C-C04 range errors on $\mathrm{C} 04$ radial biases change from 1 to 0.985 when FY-3C flies from the center to the border of the service region. The discrepancies in the range observation models caused by GEO orbit radial biases will stay almost constants since the changes of impact factors are extremely inconspicuous even though the ranges errors are very sensitive to GEO orbit radial biases. The distribution pattern of impact factors for any other GEO orbit biases is almost exactly the same as that of C04 while centering in the corresponding sub-satellite point projection on the equator. 

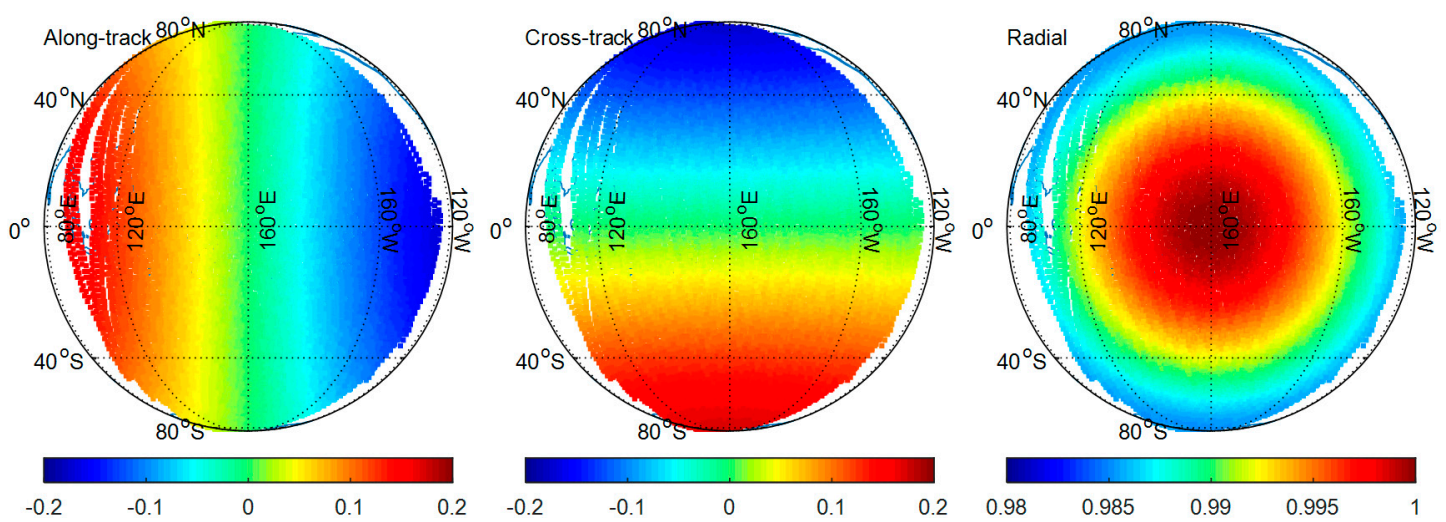

Figure 3. Magnitudes of distance errors along with the sight from FY-3C to C04 caused by unit orbit biases in the observable region for C04 during July 2017 looking down on the Earth from the perspective of $\mathrm{C} 04$. The left, center, and right are impact factors of $\mathrm{C} 04$ orbit biases in along-track, cross-track, and radial components, respectively.

In the case of FY-3C POD the GEO radial biases can be ignored since the majority of the invariant parts of the orbit radial errors and satellite clock offsets can be absorbed by the float phase ambiguity parameters. This is thanks to the small zenith angle of the line-of-sight which changes within $0^{\circ}$ to $10^{\circ}$, while biases in along-track and cross-track should be modeled accurately since the azimuth angle in the satellite orbital frame changes widely within $0^{\circ}$ to $360^{\circ}$. In view of this and the existing large DBDs that cannot be neglected in GEO orbits, the GEO orbit errors are modeled as daily systematic constant biases in the FY3C POD process.

In order to validate the performance of mitigating BeiDou GEO orbit errors using the proposed method, we made a very rough and effective experiment adopting a batch least square estimation by adjusting the receiver clock offsets and phase ambiguities while fixing the GPS-based FY-3C precise orbit in one whole day period. At the same time the weight of the observations from GEO had been set to one percent of those from IGSO and MEO. This can avoid the distortion that comes from the presence of the common parameters such as receiver clock offsets, which are contributed by observations from all BeiDou satellite types. The derived observation residuals can evaluate the performance of simulating errors in BeiDou GEO orbits as constants in along-track and cross track. Figure 4 shows the time-varying phase residual series of five GEO satellites without and with orbit biases modeled as constants in the sample period of 14 July 2017.

Figure 4 indicates that the phase residuals show apparent regular relationships with time, and the residuals range within -0.6 to $0.6 \mathrm{~m}$ without calibration of the GEO orbit biases while those decrease to -6 to $6 \mathrm{~cm}$ when calibrating the GEO orbit biases. The results indicate the efficiency of estimating the systematic biases existed in GEO orbit products together with the adjustment process while fixing the LEO reference orbit. Phase residuals with a magnitude greater than $1 \mathrm{~cm}$ are likely to be caused by limiting the weight of the phase observations from GEO to one percent with respect to those from IGSO and MEO. An assessment of more detailed phase observation residuals is carried out in Section 3.4 for FY-3C POD where all the phase observations have the same weight for different BeiDou satellite types. 


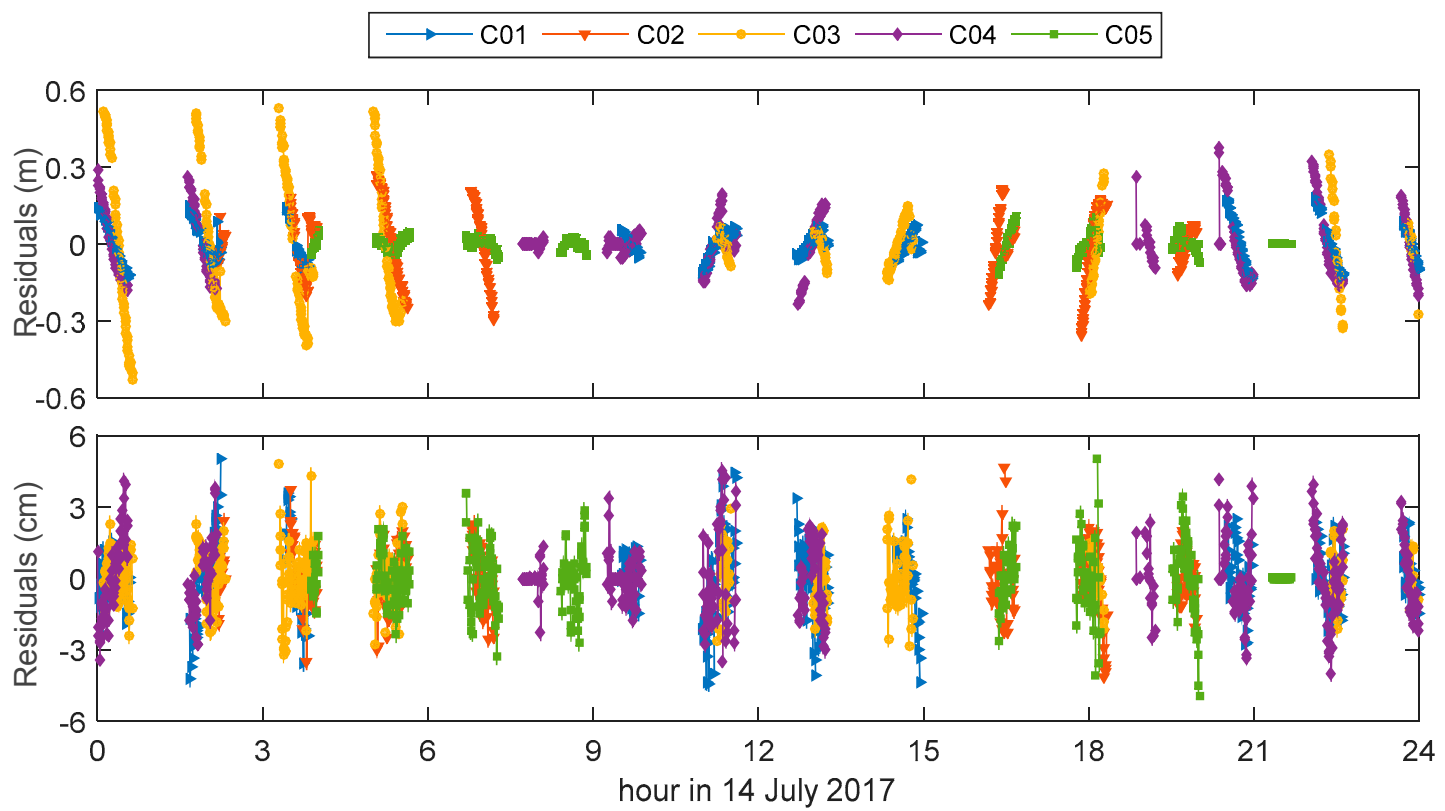

Figure 4. Phase observation residual series of five BeiDou GEO without (top) and with (bottom) calibrating orbit errors on 14 July 2017.

\section{FY-3C POD Results and Analysis}

\subsection{Strategies of $F Y-3 C P O D$}

The final precise orbit of FY-3C is generated using PANDA (Positioning And Navigation Data Analyst) software developed at GNSS Research Center of Wuhan University [27]. Each arc length of the POD is limited exactly to $24 \mathrm{~h}$ which is identical to the time period covered by daily GBM products delivered. This ensures that the orbit biases of one GEO are approximately similar, otherwise the deficient BeiDou orbits at the end-of-day of the last $5 \mathrm{~min}$ are propagated using high precision dynamic orbit fitting and integrating method. The BeiDou satellites in the attitude switch period are directly excluded in daily data processing because of their inferior orbit products.

The nominal attitude mode is used for FY-3C since the measured attitude data cannot been obtained in practice. The FY-3C satellite body fixed axes are regarded as parallel to the instantaneous orbit along-track, cross-track, and radial directions. The positioning antenna associated with the GNOS receiver is mounted on the top plane of the FY-3C body, and the antenna surface is parallel to the supporting plane. The axes of the antenna reference frame are parallel to the satellite body axes. Moreover, the origin of antenna reference frame is the antenna mounting point, the $X, Y$, and $Z$ axes in antenna frame are parallel to the $Y, X,-Z$ axes in the satellite body frame.

The ionosphere-free linear combinations of BeiDou code and phase are adopted as the basic observations. By considering the quantity of available dual-frequency BDS observation data, every six hours we estimate the atmospheric drag coefficients and the amplitudes of the periodic empirical accelerations acting on FY-3C orbit along-track and cross-track, but the empirical accelerations acting on the orbit radial are ignored. A more detailed configuration description is listed in Table 2.

Incidentally, the FY-3C reference orbit derived by onboard GPS is produced according to the strategies listed in Table 2 completely, except for the following points: The daily arc duration of POD begins at 21:00 of the previous day and ends at 03:00 of the next day, which lasts $30 \mathrm{~h}$ in order to implement the $6 \mathrm{~h}$ orbit overlap comparison, while it lasts $24 \mathrm{~h}$ for BeiDou. The FY-3C GNOS receiver positioning antenna phase center variations (PCVs) for dual-frequency GPS ionosphere-free linear combination observations have been calibrated based on the posterior phase residuals approach and corrected as the initial PCVs in the next POD process within three iterations, and the final precise orbit 
is produced in the fourth GPS-based orbit determination. However, the FY-3C PCVs for BDS are not estimated iteratively and considered as zero values in POD.

Table 2. Concepts of reduced dynamic orbit determination for FY-3C using BDS observations.

\begin{tabular}{|c|c|}
\hline Item & Description \\
\hline POD arc length & $24 \mathrm{~h}$ \\
\hline Observation selection & $\begin{array}{l}\text { Ionosphere-free linear combinations of space-borne dual-frequency phase and code } \\
\text { observations of FY-3C during July } 2017\end{array}$ \\
\hline BeiDou orbit and clock products & GBM products generated by GFZ \\
\hline BeiDou transmitter antenna phase center & Adopt antenna phase center patterns in IGS14_1967.atx [28] \\
\hline Phase wind-up & Correct using the model published in [29] \\
\hline Observation process interval & $30 \mathrm{~s}$, use observations recorded at $0 \mathrm{~s}$ and $30 \mathrm{~s}$ of each minute in GPS time system \\
\hline Cut-off elevation & Accept all available observations including those with negative elevation angles \\
\hline Observation weighting strategy & $\begin{array}{l}\text { Equal weight for phase and code respectively, and the weight ratio of phase to that of } \\
\text { code is } 40,000: 1\end{array}$ \\
\hline Earth gravity & EIGEN-6C model with degree 100 and order 100 [30] \\
\hline Solid Earth tides & IERS 2010 [31] \\
\hline Ocean tides & FES2012 model with degree 30 and order 30 [32] \\
\hline Relativistic effects & IERS 2010 \\
\hline Atmospheric drag & Box-wing model, atmospheric density adopts DTM94 model [33] \\
\hline Solar radiation pressure & Box-wing model \\
\hline Third body gravity & $\begin{array}{l}\text { Consider the gravitational attraction of celestial bodies, use celestial ephemeris named } \\
\text { DE421 published by Jet Propulsion Laboratory (JPL) [34] }\end{array}$ \\
\hline FY-3C satellite attitude & Nominal attitude mode \\
\hline Estimated parameters & $\begin{array}{l}\text { Receiver antenna phase center coordinates in } Z \text { direction estimated as a constant } \\
\text { Receiver clock offset estimated as white noise } \\
\text { Phase ambiguity estimated as a float constant during each satellite tracking arc segment } \\
\text { Satellite initial position and velocity in the inertial reference frame estimated as constants } \\
\text { Atmospheric drag coefficient estimated as piece wise constant every } 6 \mathrm{~h} \\
\text { Amplitudes of periodic empirical accelerations acting on the along-track and cross-track } \\
\text { of FY-3C orbit estimated as piece wise constants every } 6 \mathrm{~h}\end{array}$ \\
\hline
\end{tabular}

This paper designs three different POD solutions to handle the BeiDou GEO observations in FY-3C POD:

Solution A: all onboard observations of BeiDou GEO are regarded as equivalent to those of IGSO and MEO, and ignore the special errors in the GEO orbit products.

Solution B: all onboard observations of BeiDou GEO are excluded in the general POD procedure, hence the special errors in BeiDou GEO orbit products will not affect the final FY-3C orbit, but the orbit solution can be weakened because fewer observations are available.

Solution C: utilize all onboard observations of BeiDou GEO and estimate daily constant biases in orbit along-track and cross-track for each BeiDou GEO together with the POD process.

The precisions of the three different kinds of FY-3C orbits determined by solution A, B, and C respectively are assessed by comparing with the GPS-based orbit. The phase observation residuals are also analyzed to evaluate the performance of the current method for mitigating errors in BeiDou GEO orbit products.

\subsection{Distribution of FY-3C BeiDou Observations}

Dual-frequency BeiDou observation data at 30 s sampling interval measured by the positioning antenna of GNOS receiver equipped on the FY-3C satellite throughout July 2017 were used in this study. The GNOS receiver is capable of tracking pseudo-range and carrier-phase on frequency B1 and B2. The amount of available BDS data is very impressive even though the GNOS receiver can only track up to six BeiDou satellites.

Figure 5 shows the functional observation number of each BeiDou satellite and each BeiDou satellite type throughout the whole month. The right panel indicates that GEO satellites contribute 
to $32.1 \%$ of the valid observations, so it is not reasonable to exclude the GEO satellites during data processing.


Figure 5. Total number of observed times at $30 \mathrm{~s}$ interval for BeiDou satellites and types during July 2017, left subgraph represents for each BeiDou satellite and right subgraph represents for GEO, IGSO, and $\mathrm{MEO}$, respectively.

Several BeiDou satellites tracked by the FY-3C GNOS receiver on 14 July 2017 are presented in Figure 6, which illustrates the sky view containing varying patterns of elevation and azimuth for each selected satellite in the FY-3C positioning antenna reference frame. There are no apparent BeiDou signal interruptions and discontinuities [35] seen during the timeframe when observed satellites with approximately azimuth $90^{\circ}$ or $270^{\circ}$ change from the forward hemisphere to back hemisphere in the antenna view field. It is because the satellites including all range observations recorded as zero in the observation data file are also plotted in Figure 6. No obvious differences of satellite distribution can be found for BeiDou GEO, IGSO, and MEO. One tracking pass always begins with the satellite entering the field in elevation around $30^{\circ}$ and ends with the satellite disappearing when the lock on satellite signal is lost in negative elevation, which can reach a minimum of $-30^{\circ}$.
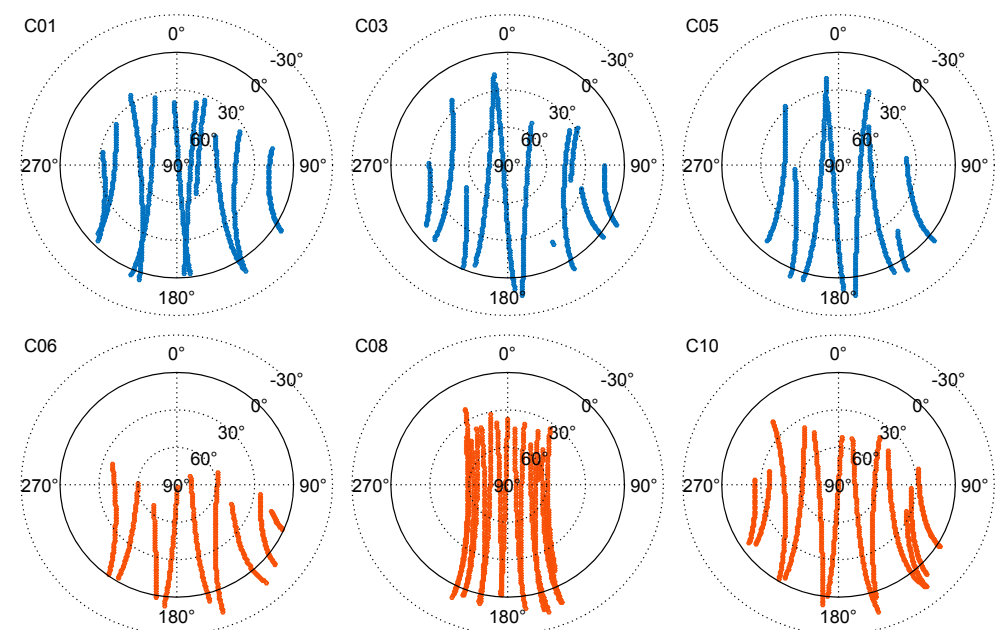

$\mathrm{C} 08$
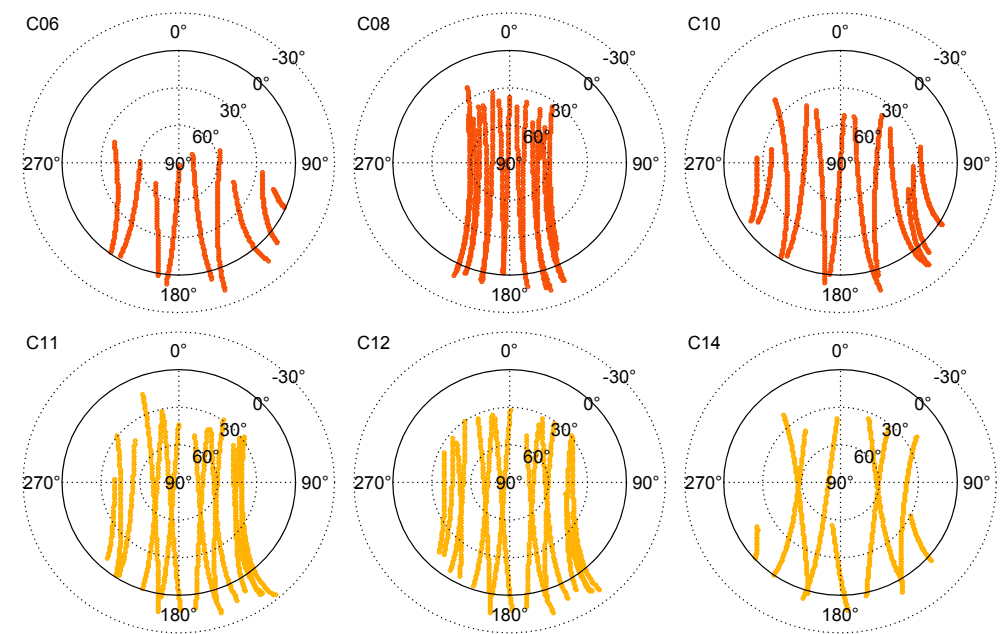

Figure 6. Sky view of BeiDou satellites in antenna reference frame observed by FY-3C GNOS receiver with 30s sampling interval on 14 July 2017. Top with blue points, middle with red points, and bottom with yellow points in the figure are prepared for GEO (C01, C03, C05), IGSO (C06, C08, C10), and MEO (C11, C12, C14), respectively. 


\subsection{Validation of FY-3C Orbit Precision}

The GPS-derived precise orbit for FY-3C is regarded as the true reference orbit to evaluate the BDS-derived orbit since the SLR data are not available. Daily RMS series of orbit differences in along-track, cross-track, radial, and 3D for the three solutions are shown in top, middle, and bottom part of Figure 7, respectively.
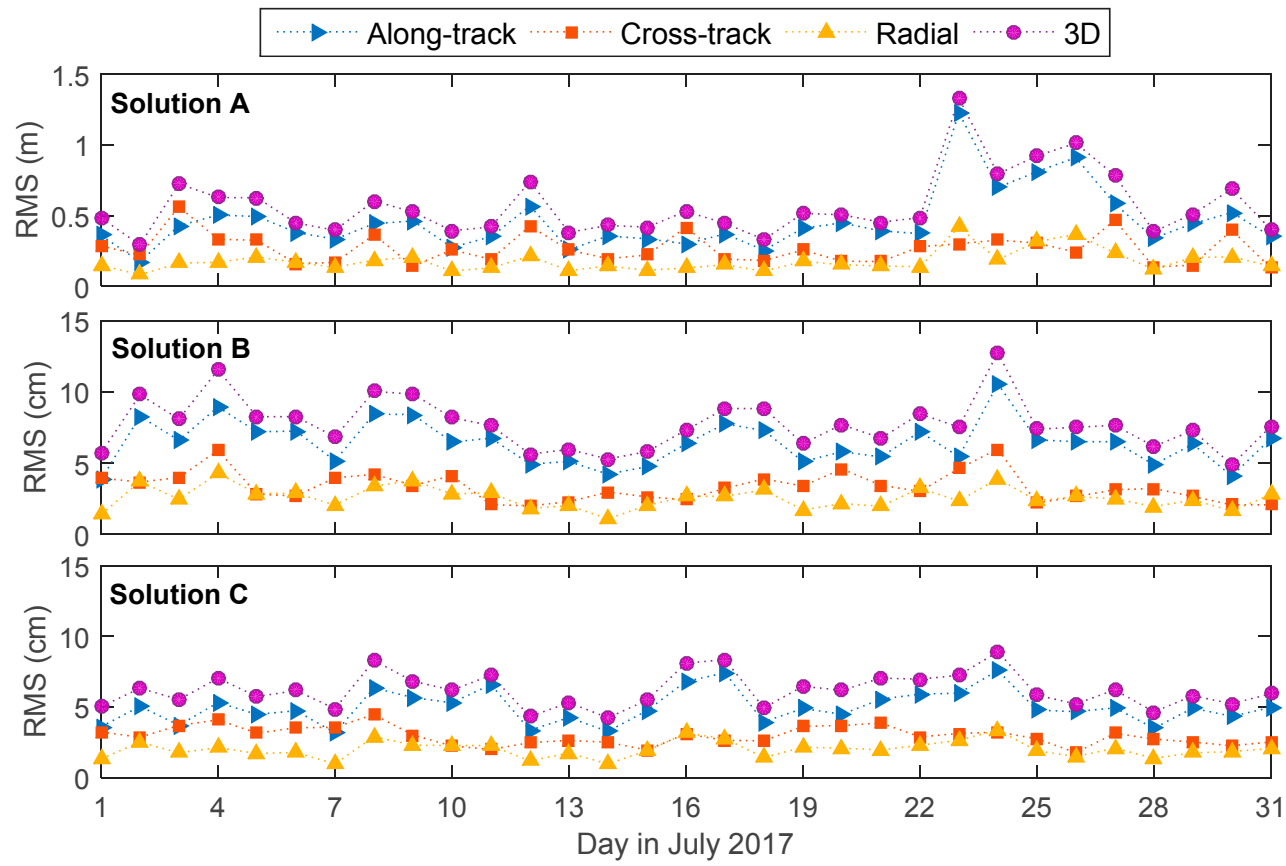

Figure 7. Daily RMS series of orbit differences in along-track, cross track, radial, and 3D between BDS-derived and GPS-derived FY-3C orbit, the top, middle, and bottom subgraphs give the solution A, $\mathrm{B}$, and $\mathrm{C}$, respectively.

The average daily RMS of orbit differences compared to GPS-derived orbit are listed in Table 3. The 3D precision of FY-3C orbit obtained from solution A is only $56.9 \mathrm{~cm}$, while the precision are 7.8 and $6.2 \mathrm{~cm}$ for solution $B$ and $C$, respectively. The orbit precision would be greatly decreased when ignoring the GEO orbit errors. The results at the centimeter level from solution $\mathrm{B}$ and $\mathrm{C}$ emphasize the significant positive contribution determined by excluding GEO and including GEO with the daily constant orbit biases calibration. A promising orbit solution for FY-3C can be achieved following the strategies of handling daily orbit biases in GEO proposed in this study. The 3D precision of the final orbit can reach $6.2 \mathrm{~cm}$ and have impressive improvements of $22.6 \%, 11.3 \%, 22.2 \%$, and $20.2 \%$ in along-track, cross-track, radial, and 3D, respectively.

Table 3. Average daily RMS of orbit differences in along-track, cross-track, radial, and 3D with three strategies for FY-3C POD using only BDS (unit: $\mathrm{cm}$ ).

\begin{tabular}{ccccc}
\hline Category & Along-Track & Cross-Track & Radial & 3D \\
\hline Solution A & 45.7 & 26.6 & 17.7 & 56.9 \\
Solution B & 6.4 & 3.3 & 2.6 & 7.8 \\
Solution C & 5.0 & 3.0 & 2.0 & 6.2 \\
\hline
\end{tabular}

\subsection{Statistics of Observation Residuals}

Residuals of the ionosphere-free linear combination for BeiDou carrier-phase observations are composite responses of receiver measurement noises, quality of BeiDou orbit and clock products, 
precision of FY-3C orbit and clock, etc. The maximum, minimum, and average values of daily RMS series of BeiDou phase observation residuals for GEO, IGSO, MEO, and all BeiDou satellites obtained from the three solutions are listed in Table 4. In Table 4 we can see that phase residuals from solution $\mathrm{B}$ and $\mathrm{C}$ are significantly smaller than those from solution $\mathrm{A}$. The residuals from solution $\mathrm{C}$ which achieves the best orbit quality are slightly larger than those from solution B.

Table 4. Maximum, minimum, and average daily RMS series of phase residuals for GEO, IGSO, MEO, and all BeiDou satellites from solution A, B, and C in FY-3C POD during July 2017 (unit: mm).

\begin{tabular}{ccccc}
\hline Category & $\begin{array}{c}\text { GEO } \\
\text { Max/Min/Mean }\end{array}$ & $\begin{array}{c}\text { IGSO } \\
\text { Max/Min/Mean }\end{array}$ & $\begin{array}{c}\text { MEO } \\
\text { Max/Min/Mean }\end{array}$ & $\begin{array}{c}\text { BeiDou } \\
\text { Max/Min/Mean }\end{array}$ \\
\hline Solution A & $126.0 / 35.4 / 63.5$ & $71.0 / 21.9 / 36.5$ & $47.4 / 18.9 / 29.8$ & $73.1 / 28.4 / 45.8$ \\
Solution B & - & $7.1 / 5.0 / 6.2$ & $7.2 / 4.5 / 6.2$ & $7.1 / 4.8 / 6.2$ \\
Solution C & $10.1 / 8.0 / 8.9$ & $8.6 / 7.0 / 7.5$ & $7.8 / 6.1 / 7.1$ & $8.5 / 7.3 / 7.9$ \\
\hline
\end{tabular}

Average daily RMS of BDS phase residuals are 45.8, 6.2, and $7.9 \mathrm{~mm}$ for solution A, B, and C, respectively. The average daily RMS of residuals derived in solution $B$ and solution $C$ are all less than one centimeter. This demonstrates a good matching attribute between the BeiDou observation models and the FY-3C orbit dynamic models. Residuals at the centimeter level obtained in solution C also prove that it is a practical method for eliminating most of the errors in BeiDou GEO orbit products by modeling systematic errors as daily constants in along-track and cross-track of the GEO orbits. The results are consistent with the precision evaluation of calibrated GEO orbits in Section 3.5.

The residuals derived in solution B and C should be compared and analyzed much more meticulously since they both obtained one centimeter level residuals while solution A achieved half-decimeter level residuals. Figure 8 shows the daily RMS values of phase residuals for GEO, IGSO, MEO, and overall BeiDou satellites in FY-3C POD for solution B and C during July 2017. Both Figure 8 and Table 4 indicate that the daily RMS of GEO phase residuals mostly range between 8 and $10 \mathrm{~mm}$ when the GEO orbit biases are in-flight calibrated correctly in LEO POD. Daily RMS of IGSO and MEO mostly range between 5 and $9 \mathrm{~mm}$ for solution $\mathrm{B}$ and $\mathrm{C}$. In addition, the RMS values of IGSO and MEO for solution C are slightly larger than those for solution B.

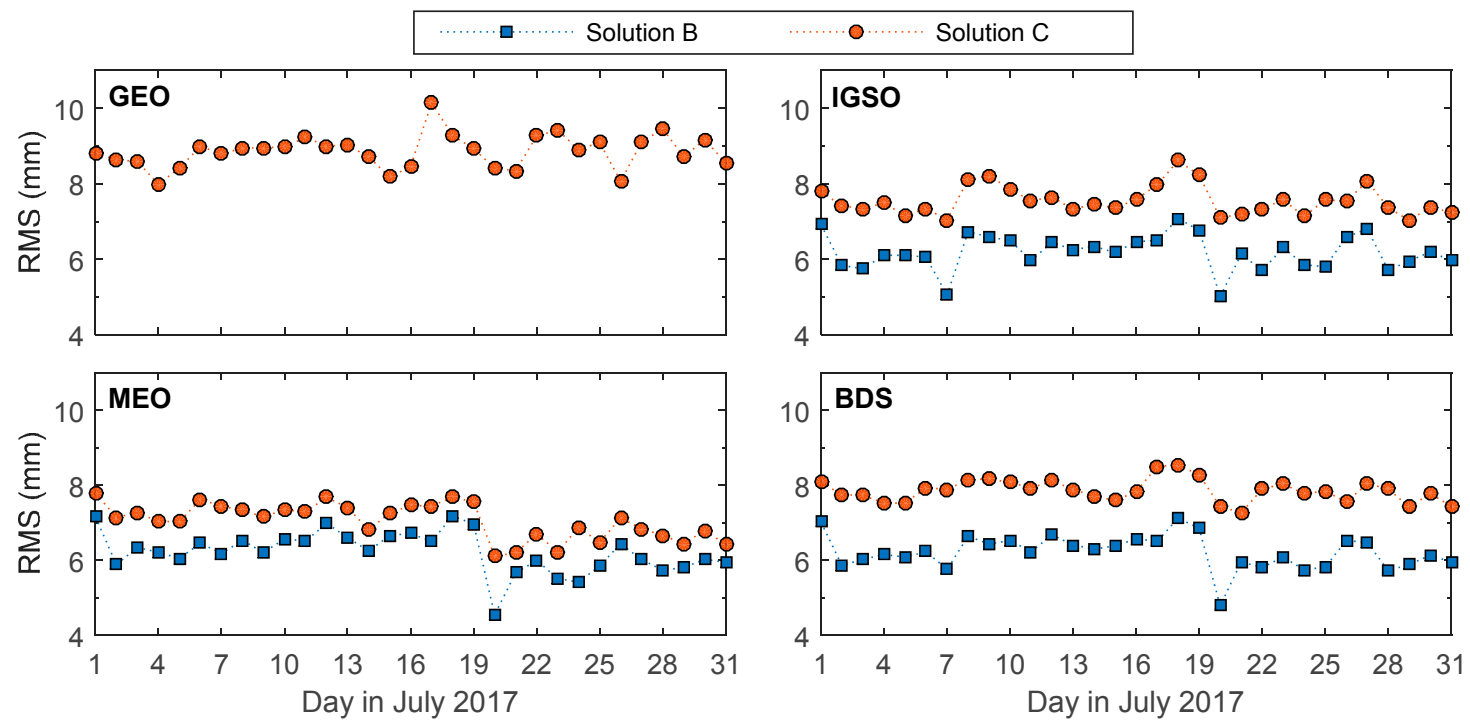

Figure 8. Daily RMS series of phase observation residuals for GEO, IGSO, MEO, and all BeiDou satellites in POD of FY-3C with different solution B and C. The blue squares and red circles represent the daily RMS of residuals obtained in solution B and C, respectively. The upper left, upper right, lower left, and lower right denote for GEO, IGSO, MEO, and all BeiDou satellites, respectively. 
Several reasons can explain the phenomenon that the daily RMS series of phase residuals from solution B are slightly less than those from solution C. First, stronger constraints in the BeiDou observation models could be achieved with an increase in the redundant observations of solution $\mathrm{C}$ containing GEO compared to those of Solution B. Second, observations are contaminated by GEO orbit and clock errors that cannot be modeled completely by using the calibration method of constant biases existing in along-track and cross-track of GEO orbits. Third, the quality of the BeiDou GEO products cannot reach the same accuracy level with respect to that of IGSO and MEO even though the systematic errors existing in GEO orbits can be estimated completely. It should be noted that the orbit quality of solution $C$ is the best even though the average daily RMS of the residuals is slightly larger than that of solution B by about $1.7 \mathrm{~mm}$.

\subsection{Assessment of Calibrated BeiDou GEO Orbits}

The daily constant biases in the along-track and cross-track of BeiDou GEO orbit products can be estimated in the LEO POD procedure. Figure 9 shows the calibrated daily biases in GBM BeiDou GEO orbit products during July 2017. All the orbit biases are in several meters and the maximum bias does not exceed $8 \mathrm{~m}$. The orbit biases change every day but hardly show any obvious regularities, and biases vary for different BeiDou GEO satellites. The overall magnitudes of BeiDou GEO orbit biases in the along-track are slightly larger than those in the cross-track except the C04 satellite. The values of estimated daily orbit biases in the BeiDou GEO presented here are only available in the case of GBM products generated by GFZ. The biases in other BeiDou GEO orbit products are vastly different since other ACs compute the BeiDou orbit products generally adopting dissimilar ground stations, observation models, and force modes, etc.


Figure 9. Estimated daily systematic orbit biases in along-track and cross-track of GBM BeiDou GEO orbit products published by GFZ during July 2017 in FY-3C POD. The left and right subgraphs denote the daily orbit bias series in the BeiDou GEO orbit along-track and cross-track, respectively. Different color points are prepared for different GEO satellites.

The calibrated orbits of GEO can be recovered through applying the estimated daily constant biases in along-track and cross-track to the original fitted orbits, the DBDs of the reconstructed orbits are adopted to assess the performance of GEO orbit errors in-flight calibration method. Since there are irregular DBDs with several meters in calibrated orbits, the RMS involving all DBDs and the RMS excluding the DBDs more than $1 \mathrm{~m}$ in along-track or cross-track are used to assess the quality of calibrated orbits. The DBDs of the original fitted orbits are also rejected if the corresponding DBDs of the calibrated orbits are excluded. Figure 10 shows the RMS of DBDs of the original and calibrated orbits by excluding anomalous DBDs for each BeiDou GEO satellite. The abnormal DBDs occur synchronously in the original and calibrated orbits especially in along-track of the C04 and C05 orbits. The irregular DBDs are caused by the large DBDs existing in the original GEO orbits. This means that 
the estimated GEO orbit biases are not correct when the DBDs of the original GEO orbits are large or irregular.

When the outliers in DBDs are removed, the average RMS of DBDs in the calibrated orbits are decreased to $0.29 \mathrm{~m}$ and $0.21 \mathrm{~m}$, which give improvements of $78.4 \%$ and $83.9 \%$ with respect to those in the original fitted orbits in along-track and cross-track, respectively. The results indicate that the GEO orbits at the decimeter level in along-track and cross-track can be recovered by correcting the estimated GEO orbit systematic errors in FY-3C POD. The calibrated GEO orbits can be considered as another form of enhanced orbits which can be used to perform POD for other LEO using BeiDou GEO observations.


Figure 10. RMS of DBDs in along-track and cross-track for each BeiDou GEO satellite of the original fitted orbits and calibrated orbits with biases correction, top part of the figure denotes the RMS of all DBDs participate in statistics, the bottom part denotes the RMS by excluding the DBDs with more than $1 \mathrm{~m}$ in along-track or cross-track for the calibrated orbits.

\section{Discussion}

The reason that systematic biases frequently appear in BeiDou GEO orbit products can be explained by diagnosing the almost unchanged geometric observation condition in the current BeiDou POD strategy which is based purely on the ground tracking station network. Effects of constant offsets in GEO orbits can also be eliminated in the PPP of low kinematic stations in accordance with the same idea. However, the effects in the POD of LEO cannot be removed directly because of varying range errors along with the rapidly changing of line-of-sight between LEO and GEO. We attempt to estimate the orbit systematic biases of GEO in orbital reference frame as well as the receiver clock errors and the phase ambiguity parameters by using onboard BDS measurements when fixing the GPS-derived precise orbit of FY-3C. The results indicate that in-flight calibration of systematic biases in GEO orbit products is feasible and effective in view of the posteriori phase observation residuals, which reduce from decimeter level to several centimeters under the constraints of reference precise FY-3C orbit.

Even though the results in this article exhibit a positive contribution from involving GEO on FY-3C POD using BDS observations, there are still several associated aspects that need to be studied with more detailed investigations. Characteristics of systematic biases in BeiDou GEO products should be modeled carefully using comprehensive patterns instead of the simple daily constants in along-track and cross-track currently adopted. The daily jumps in the GEO orbit systematic biases cannot be neglected since they are caused by the orbit DBDs and can reach several meters. Moreover, they should be taken into a special consideration so it is possible to realize the POD for a long arc beyond $24 \mathrm{~h}$ and 
obtain more reasonable reduced dynamic orbit solution. Estimated frequencies, priori constraints, and mathematical models, etc. of the coefficients associated with non-conservative forces and empirical accelerations deserve a thorough optimization to reach a favorable balance between the observation level with current BeiDou products and LEO orbit dynamics. Estimation of the variance component for observations from different BeiDou satellite types, such as GEO, IGSO, and GEO may probably perform an important function in the POD relative to the fully-equivalent weight for all observations. The PCV map in BeiDou signal frequencies of the positioning antenna connected to the FY-3C GNOS receiver should be calibrated using onboard BDS observations along with the POD process in order to achieve a better orbit solution.

The well-performed processing strategy of calibrating daily orbit biases that we recommend for FY-3C POD using BDS observations does not only apply to BeiDou GEO, but also to any other satellites which have comparable systematic errors in orbits. At the same time, the method used to absorb orbit errors currently existing in BeiDou GEO is temporary at this stage because GEO products are produced using only static ground stations. Calibration of GEO orbit biases in LEO POD will be no longer needed if the GEO products are enhanced using observations collected by highly-kinematic stations such as the LEO, vessel, balloon, airship, plane, and so forth. It would be a tremendous opportunity and challenge for the integrated POD of BeiDou and other navigation satellite constellations using all available ground, space-borne, and onboard data from multiple sensors and multiple platforms. We will investigate more interesting and meaningful analysis in the not-so-distant future.

\section{Conclusions}

The poor orbit accuracy of BeiDou GEO satellites is the major challenge to BDS-based LEO POD. In this study we proposed a GEO orbit bias in-flight calibration algorithm which mitigates the adverse impacts of the BeiDou GEO orbit biases on the LEO POD. When compared to the algorithm for neglecting GEO orbit biases completely and the algorithm for excluding the GEO roughly, the proposed algorithm enables the LEO POD to benefit from the BeiDou GEO observations. In our algorithm the along-track and cross-track GEO orbit biases are estimated as daily constants and the radial orbit biases are neglected. The efficiency of the algorithm is validated by one month of space-borne BDS observations from the FY-3C satellite. The FY-3C satellite allows us to conduct a highly-significant analysis of the FY-3C reduced dynamic POD using only BDS and the contribution of BeiDou GEO to POD with and without the calibration of orbit systematic errors.

We investigated the effects and contribution of involving GEO in the POD of FY-3C by comparing three strategies: disregarding GEO orbit errors, excluding GEO, and calibrating GEO orbit biases. The obtained orbit using all observations from three types of BeiDou satellites shows an orbit precision of $0.57 \mathrm{~m}$ in average 3D RMS when compared to the GPS-derived orbit. Hence, it is not possible to achieve a centimeter level precise orbit with current orbit products of BeiDou GEO. A precision of $7.8 \mathrm{~cm}$ in $3 \mathrm{D}$ achieved for the FY-3C orbit demonstrates a significant improvement of $86.4 \%$ when the observations of GEO are rejected. More available observations can enhance the performance in the POD of FY-3C by including BeiDou GEO and the simultaneous calibration of systematic biases in GEO orbit products. The precision of the obtained orbit reaches an encouraging level of $6.2 \mathrm{~cm}$ in 3D, which represents a general improvement of $20.2 \%$ with respect to that obtained by excluding GEO. The results are further confirmed by the residual analysis according to the average daily RMS of the posteriori phase residuals are $45.8,6.2$, and $7.9 \mathrm{~mm}$ for the above three solutions, respectively. The RMS of DBDs in the recovered GEO orbits with daily orbit biases calibrated using FY-3C onboard BDS data are also used to assess the performance of the proposed method. If the abnormal DBDs in the calibrated orbits are removed, the average RMS of DBDs for GEO decrease to $0.29 \mathrm{~m}$ and $0.21 \mathrm{~m}$ in along-track and cross-track, respectively. The calibrated GEO orbits can be directly used to perform POD for other LEO satellites.

The precise orbit of FY-3C is obtained using BDS data with estimation of daily systematic biases existing in GEO orbit products in this investigation. It remains a progressive achievement for the 
current BeiDou navigation constellation which provides service focused mainly on the Asia-Pacific region. There is no doubt that the performance of the BDS-based POD of LEO could reach a comparable level to GPS with more and more BeiDou satellites launched and gradually improving quality of BeiDou products.

Acknowledgments: We are greatly appreciative to the GFZ and WHU analysis center of MGEX for providing BeiDou precise orbit and clock products. This research work is sponsored by the Natural Science Foundation of China (grant no. 41774035).

Author Contributions: Qiang Zhang conceived, designed, and performed the experiments; Xiang Guo analyzed the onboard GPS and BDS data from FY-3C GNOS receiver; Lizhong Qu and Qile Zhao contributed software packages for precision orbit determination of LEO satellites; and Qiang Zhang wrote the paper.

Conflicts of Interest: The authors declare no conflict of interest.

\section{References}

1. Kang, Z.G.; Tapley, B.; Bettadpur, S.; Ries, J.; Nagel, P.; Pastor, R. Precise orbit determination for the GRACE mission using only GPS data. J. Geodesy 2006, 80, 322-331. [CrossRef]

2. Bruce, H.; Yoaz, B.; Willy, B.; Shailen, D.; Pascal, W. One-Centimeter Orbit Determination for Jason-1: New GPS-Based Strategies. Mar. Geodesy 2004, 27, 299-318.

3. Bock, H.; Jäggi, A.; Meyer, U.; Visser, P.; van den Ijssel, J.; van Helleputte, T.; Heinze, M.; Hugentobler, U. GPS-derived orbits for the GOCE satellite. J. Geodesy 2011, 85, 807-818. [CrossRef]

4. Bock, H.; Jäggi, A.; Švehla, D.; Beutler, G.; Hugentobler, U.; Visser, P. Precise orbit determination for the GOCE satellite using GPS. Adv. Space Res. 2007, 39, 1638-1647. [CrossRef]

5. Van den IJssel, J.; Encarnacao, J.; Doornbos, E.; Visser, P. Precise science orbits for the Swarm satellite constellation. Adv. Space Res. 2015, 56, 1042-1055. [CrossRef]

6. Guo, J.; Zhao, X.; Liu, X.; Liu, J.; Zhou, Q. Quality assessment of onboard GPS receiver and its combination with DORIS and SLR for Haiyang 2A precise orbit determination. Sci. China Earth Sci. 2015, 138-150. [CrossRef]

7. Min, L.; Qu, L.; Zhao, Q.; Jing, G.; Xing, S.; Li, X. Precise Point Positioning with the BeiDou Navigation Satellite System. Sensors 2014, 14, 927-943.

8. Chen, X.; Zhao, S.; Wang, M.; Lu, M. Space-borne BDS receiver for LING QIAO satellite: Design, implementation and preliminary in-orbit experiment results. GPS Solut. 2015, 20, 837-847. [CrossRef]

9. Zhao, S.; Chen, X.; Yuan, S.; Kuang, L.; Lu, M. Space-borne BDS and GPS Receiver of LING QIAO: Results over Two Years In-Orbit Operation. In Proceedings of the China Satellite Navigation Conference, Shanghai, China, 23-25 May 2017; pp. 753-762.

10. Liu, J.H.; Gu, D.F.; Ju, B.; Yao, J.; Duan, X.J.; Yi, D.Y. Basic performance of BeiDou-2 navigation satellite system used in LEO satellites precise orbit determination. Chin. J. Aeronaut. 2014, 27, 1251-1258. [CrossRef]

11. Xiong, C.; Lu, C.F.; Zhu, J.; Ding, H.P. Orbit determination using real tracking data from FY3C-GNOS. Adv. Space Res. 2017, 60, 543-556. [CrossRef]

12. Bi, Y.M.; Yang, Z.D.; Zhang, P.; Sun, Y.Q.; Bai, W.H.; Du, Q.F.; Yang, G.L.; Chen, J.; Liao, M. An introduction to China FY3 radio occultation mission and its measurement simulation. Adv. Space Res. 2012, 49, 1191-1197. [CrossRef]

13. Bai, W.H.; Sun, Y.Q.; Du, Q.F.; Yang, G.L.; Yang, Z.D.; Zhang, P.; Bi, Y.M.; Wang, X.Y.; Cheng, C.; Han, Y. An introduction to the FY3 GNOS instrument and mountain-top tests. Atmos. Meas. Tech. 2014, 7, 1817-1823. [CrossRef]

14. Cai, Y.; Bai, W.; Wang, X.; Sun, Y.; Du, Q.; Zhao, D.; Meng, X.; Liu, C.; Xia, J.; Wang, D.; et al. In-orbit performance of GNOS on-board FY3-C and the enhancements for FY3-D satellite. Adv. Space Res. 2017, 60, 2812-2821. [CrossRef]

15. Li, M.; Li, W.W.; Shi, C.; Jiang, K.C.; Guo, X.; Dai, X.L.; Meng, X.G.; Yang, Z.D.; Yang, G.L.; Liao, M. Precise orbit determination of the Fengyun-3C satellite using onboard GPS and BDS observations. J. Geodesy 2017, 91, 1313-1327. [CrossRef]

16. Zhao, Q.L.; Wang, C.; Guo, J.; Yang, G.L.; Liao, M.; Ma, H.Y.; Liu, J.N. Enhanced orbit determination for BeiDou satellites with FengYun-3C onboard GNSS data. GPS Solut. 2017, 21, 1179-1190. [CrossRef] 
17. Palmerini, G. Capabilities of the GNSS Precise Point Positioning Technique for Landslide Monitoring. Disaster Adv. 2012, 5, 509-513.

18. Kouba, J.; Héroux, P. Precise Point Positioning Using IGS Orbit and Clock Products. GPS Solut. 2001, 5, 12-28. [CrossRef]

19. Zhao, Q.; Wang, C.; Guo, J.; Liu, X. Assessment of the Contribution of BeiDou GEO, IGSO, and MEO Satellites to PPP in Asia-Pacific Region. Sensors 2015, 15, 29970-29983. [CrossRef] [PubMed]

20. Montenbruck, O.; Steigenberger, P.; Prange, L.; Deng, Z.G.; Zhao, Q.L.; Perosanz, F.; Romero, I.; Noll, C.; Sturze, A.; Weber, G.; et al. The Multi-GNSS Experiment (MGEX) of the International GNSS Service (IGS)-Achievements, prospects and challenges. Adv. Space Res. 2017, 59, 1671-1697. [CrossRef]

21. Dow, J.M.; Neilan, R.E.; Rizos, C. The International GNSS Service in a changing landscape of Global Navigation Satellite Systems. J. Geodesy 2009, 83, 191-198. [CrossRef]

22. Uhlemann, M.; Gendt, G.; Ramatschi, M.; Deng, Z. GFZ Global Multi-GNSS Network and Data Processing Results. In IAG 150 Years, Proceedings of the IAG Scientific Assembly, Postdam, Germany, 1-6 September 2016; Rizos, C., Willis, P., Eds.; Springer International Publishing: Cham, Switzerland, 2016; pp. 673-679.

23. Guo, F.; Li, X.; Zhang, X.; Wang, J. Assessment of precise orbit and clock products for Galileo, BeiDou, and QZSS from IGS Multi-GNSS Experiment (MGEX). GPS Solut. 2017, 21, 279-290. [CrossRef]

24. Guo, J.; Xu, X.; Zhao, Q.; Liu, J. Precise orbit determination for quad-constellation satellites at Wuhan University: Strategy, result validation, and comparison. J. Geodesy 2016, 90, 143-159. [CrossRef]

25. Lou, Y.; Zhang, W.; Wang, C.; Yao, X.; Shi, C.; Liu, J. The impact of orbital errors on the estimation of satellite clock errors and PPP. Adv. Space Res. 2014, 54, 1571-1580. [CrossRef]

26. Douša, J. The impact of errors in predicted GPS orbits on zenith troposphere delay estimation. GPS Solut. 2010, 14, 229-239. [CrossRef]

27. Liu, J.N.; Mao-Rong, G.E. Panda Software and Its Preliminary Result of Positioning and Orbit Determination. Wuhan Univ. J. Nat. Sci. 2003, 8, 603-609.

28. Rebischung, P.; Schmid, R. IGS14/igs14.atx: A New Framework for the IGS Products. In Proceedings of the AGU Fall Meeting, San Francisco, CA, USA, 12-16 December 2016.

29. Wu, J.T.; Wu, S.C.; Hajj, G.A.; Bertiger, W.I.; Lichten, S.M. Effects of Antenna Orientation on GPS Carrier Phase. In Proceedings of the AAS/AIAA Astrodynamics Conference; Durango, CO, USA, 19-22 August 1991; pp. 1647-1660.

30. Shako, R.; Förste, C.; Abrikosov, O.; Bruinsma, S.; Marty, J.-C.; Lemoine, J.-M.; Flechtner, F.; Neumayer, H.; Dahle, C. EIGEN-6C: A High-Resolution Global Gravity Combination Model Including GOCE Data. In Observation of the System Earth from Space-Champ, Grace, Goce and Future Missions: Geotechnologien Science Report No. 20; Flechtner, F., Sneeuw, N., Schuh, W.-D., Eds.; Springer: Berlin/Heidelberg, Germany, 2014; pp. 155-161.

31. Petit, G.; Luzum, B.; Al, E. IERS Conventions. IERS Tech. Note 2010, 36, 1-95.

32. Carrere, L.; Lyard, F.; Cancet, M. FES 2012: A New Global Tidal Model Taking Advantage of Nearly 20 Years of Altimetry. In Proceedings of the 20 Years of Progress in Radar Altimatry, Venice, Italy, 24-29 September 2013; Volume 710, pp. 11-12.

33. Berger, C.; Biancale, R.; IllF, M. Barlier Improvement of the empirical thermospheric model DTM: DTM94-A comparative review of various temporal variations and prospects in space geodesy applications. J. Geodesy 1998, 72, 161-179. [CrossRef]

34. Folkner, W.M.; Williams, J.G.; Boggs, D.H. The Planetary and Lunar Ephemeris DE 421. Interplanet. Netw. Prog. Rep. 2009, 178, 1-34.

35. Jiang, K.; Li, M.; Zhao, Q.; Li, W.; Guo, X. BeiDou Geostationary Satellite Code Bias Modeling Using Fengyun-3C Onboard Measurements. Sensors 2017, 17, 2460. [CrossRef] [PubMed]

(C) 2018 by the authors. Licensee MDPI, Basel, Switzerland. This article is an open access article distributed under the terms and conditions of the Creative Commons Attribution (CC BY) license (http:/ / creativecommons.org/licenses/by/4.0/). 